\title{
Taxa de prenhez em vacas de leite após uso de protocolos hormonais de inseminação artificial em tempo fixo*
}

\section{Pregnancy rates in dairy cows after the use of hormonal protocols of timed artificial insemination}

\author{
Ítalo Câmara de Almeida, ${ }^{* *}$ Tiago Botelho Gomes, ${ }^{* * *}$ Mateus Moreira de Pinho, ${ }^{* * *}$ João Gomes de Siqueira, ${ }^{* * * *}$ \\ Larissa Marchiori Sena, ${ }^{* * * * *}$ Carlos Augusto de Alencar Fontes ${ }^{* * * * * * *}$
}

\begin{abstract}
Resumo
O presente estudo objetivou comparar a eficiência e o custo de três protocolos de inseminação artificial em tempo fixo (IATF), em vacas leiteiras, criadas em sistema extensivo de produção nas regiões Norte e Noroeste Fluminense. Foram utilizadas 162 vacas mestiças Bos taurus taurus x Bos taurus indicus, hígidas, avaliadas previamente por exame de ultrassonografia. Foi utilizado sêmen comercial de diferentes touros devidamente testados nas centrais de inseminação. As vacas receberam implantes intravaginais de progesterona e foram submetidas aos protocolos: BE $(n=108)$ - benzoato de estradiol; $\mathrm{GnRH}(n=22)$ - benzoato de estradiol e hormônio liberador de gonadotrofinas $(\mathrm{GnRH})$; e eCG $(n=32)$ - benzoato de estradiol, GnRH e gonadotrofina coriônica equina (eCG). Decorridos 45 dias da IATF foi realizado exame de ultrassonografia para a detecção da gestação. Os dados foram analisados por meio do teste de qui-quadrado. As vacas submetidas aos protocolos BE, GnRH e eCG obtiveram os índices de prenhez 42,6\%, 45,5\% e 68,8\% respectivamente. O protocolo que apresentou menor custo econômico foi o BE, seguido do eCG e por último o GnRH. O uso da IATF na região pode ser alternativa viável para incrementar os índices reprodutivos em rebanhos bovinos, sobretudo com uso de protocolos hormonais que tenham em sua composição a eCG que, segundo o presente estudo, apresentaram índices de prenhes satisfatórios e custo econômico mediano.
\end{abstract}

Palavras-chave: biotécnicas reprodutivas, eficiência reprodutiva, IATF, reprodução animal, vacas mestiças.

\begin{abstract}
This study aimed to compare the efficiency and the cost of three protocols of timed artificial insemination (TAI) in dairy cows reared in extensive production system in the North and Northwest Fluminense regions. They used 162 crossbred cows Bos taurus taurus $x$ Bos taurus indicus, otherwise healthy, previously assessed by ultrasound examination. It used commercial semen from different bulls properly tested in insemination centers. The cows received implants intravaginal progesterone and were submitted to the protocols: $\mathrm{BE}(\mathrm{n}=108)$ - estradiol benzoate; $\mathrm{GnRH}(\mathrm{n}=22)$ - estradiol benzoate and gonadotropin-releasing hormone $(\mathrm{G} n \mathrm{RH})$; and eCG ( $n=32$ ) - estradiol benzoate, GnRH and equine chorionic gonadotropin (eCG). After 45 days of TAI was performed ultrasound examination for the detection of pregnancy. Data were analyzed using the chi-square test. The cows submitted to BE protocols, $\mathrm{GnRH}$ and eCG obtained the pregnancy rates $42.6 \%, 45.5 \%$ and $68.8 \%$ respectively. The protocol showed lower economic cost was the $\mathrm{BE}$, followed by eCG and last GnRH. The use of the TAl in the region can be a viable alternative to increase the reproductive rates in cattle herds, especially with the use of hormonal protocols that have in their composition eCG that, according to this study showed satisfactory pregnant rates and average economic cost.
\end{abstract}

Keywords: animal reproduction, crossbred cows, reproductive biotechnologies, reproductive efficiency, TAI.

\section{Introdução}

Embora seja um dos maiores consumidores de produtos lácteos do país (cerca de 3,2 bilhões de toneladas/ano), o estado do Rio de Janeiro tem produção pouco expressiva de leite (aproximadamente 489 milhões de litros/ano), o que não é suficiente para atender a demanda de um quinto dos consumidores. No entanto, a produção leiteira fluminense está em franca expansão e movimenta uma renda de aproximadamente
$\mathrm{R} \$ 6,4$ bilhões anualmente (Colbert, 2012). Apesar das regiões Norte e Noroeste Fluminense serem as microbacias leiteiras do estado com maior volume de leite produzido por área, existem fatores limitantes que dificultam a obtenção de melhores resultados, como a baixa produtividade e qualidade genética de seus rebanhos (FAERJ/SEBRAE-RJ, 2003).

A inserção de biotecnologias reprodutivas na pecuária leiteira vem alavancando a produtividade do setor, aumentando a

*Recebido em 17 de maio de 2015 e aceito em 2 de julho de 2016.

${ }^{* *}$ M. V., Mestre em Ciências Veterinárias e Auxiliar de Veterinária e Zootecnia do Departamento de Medicina Veterinária da Universidade Federal do Espírito Santo; Alegre-ES, Brasil. Autor para correspondência: almeidaicvet@gmail.com.

***M. V., Mestre em Ciência Animal pela Universidade Estadual do Norte Fluminense Darcy Ribeiro; Campos dos Goytacazes-RJ, Brasil.

****M. V., pela Universidade Estadual do Norte Fluminense Darcy Ribeiro; Campos dos Goytacazes-RJ, Brasil.

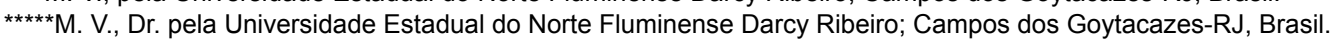

******Mestranda em Ciências Veterinárias; Departamento de Medicina Veterinária da Universidade Federal do Espírito Santo; Alegre-ES, Brasil.

${ }_{* * * * * * *}$ Docente do Centro de Ciências Agrárias da Universidade Estadual do Norte Fluminense Darcy Ribeiro; Campos dos Goytacazes-RJ, Brasil. 
renda dos produtores, proporcionando melhoramento genético do rebanho, aumentando o número de parições e diminuindo o intervalo de partos. Em conjunto com a utilização destas biotecnologias reprodutivas, há outros fatores de fundamental importância para o aumento da produtividade do rebanho, como: sanidade, nutrição e condição corporal, lactação e manejo produtivo (Montezuma Jr., 2001; Ferreira, 2000).

Nota-se grande complexidade em torno da pecuária leiteira para que se possa alcançar boa eficiência reprodutiva do rebanho. Entretanto, biotecnologias reprodutivas como a inseminação artificial em tempo fixo (IATF) permitem a parição estratégica das vacas, a formação de lotes padronizados e homogêneos, a redução da ocorrência de problemas reprodutivos e a melhoria da qualidade genética dos animais (Sá Filho et al., 2006).

Tendo em vista o numeroso rebanho que compõe as regiões Norte e Noroeste Fluminense e a importância da bovinocultura na economia dessas regiões, verifica-se a necessidade da realização de estudos a respeito da utilização da IATF para que se obtenha maior eficiência e, consequentemente, viabilidade econômica da mesma. Objetivou-se com o presente estudo avaliar o uso de três diferentes protocolos hormonais em vacas leiteiras das regiões Norte e Noroeste Fluminense para a realização da IATF, comparando a eficácia dos protocolos sobre a taxa de gestação e o custo por prenhez em cada protocolo utilizado.

\section{Material e métodos}

O estudo foi realizado de outubro de 2012 a março de $2013 \mathrm{em}$ propriedades rurais das regiões Norte e Noroeste Fluminense que têm como atividade principal a produção leiteira, nos municípios de Campos dos Goytacazes, Conceição de Macabu e Bom Jesus de Itabapoana. Em todas as propriedades as vacas eram mantidas em sistema extensivo de produção, com pastagem composta principalmente de Brachiaria sp. Sal mineral e água eram fornecidos ad libitum. Os animais utilizados foram vacas mestiças Bos taurus taurus $\times$ Bos taurus indicus com diferentes graus de sangue, ordem de parição, produtividade média de 10L/vaca/dia e média de 55,6 $\pm 7,8$ dias de pós-parto.

Antes do início de cada protocolo as vacas foram selecionadas através de exame sanitário e ginecológico com o uso da ultrassonografia, para que a sincronização fosse feita somente naquelas consideradas aptas. Vacas com qualquer distúrbio metabólico, reprodutivo e/ou histórico de infertilidade não foram incluídas e apenas as negativas para brucelose e tuberculose foram utilizadas. Apenas fêmeas com ECC $\geq 2,5$ (escala de 1 a 5; Edminson et al. 1989), foram incluídas no experimento. As vacas selecionadas $(n=162)$, receberam um dos protocolos hormonais comumente utilizados em programas de IATF:

- Protocolo BE ( $n=108)$ : no dia zero (D0), as fêmeas receberam dispositivos intravaginais de progesterona $\left(D^{1} P^{1}\right)$ novo e aplicação intramuscular (i.m.) de $2 \mathrm{mg}$ benzoato de estradiol $\left(\mathrm{BE}^{2}\right)$; em D8, foi feita a retirada do DIP e aplicou-se $0,15 \mathrm{mg}$ de cloprostenol sódico (Prostaglandina') i.m.; em D9, aplicou-se 1 $\mathrm{mg}$ de BE i.m. e em; D10, procedeu-se a inseminação artificial (IA) dos animais às 52 horas após a retirada do DIP.

${ }^{1}$ Sincrogest ${ }^{\circledR}$, Ouro Fino Saúde Animal, Cravinhos, São Paulo, Brasil

${ }^{2}$ Sincrodiol ${ }^{\circledR}$, Ouro Fino Saúde Animal, Cravinhos, São Paulo, Brasil;
- Protocolo $\mathrm{GnRH}$ (n=22): no D0 as fêmeas receberam DIP novo e aplicação i.m. de $2 \mathrm{mg} \mathrm{BE;} \mathrm{em} \mathrm{D8,} \mathrm{foi} \mathrm{feita} \mathrm{a} \mathrm{retirada} \mathrm{do} \mathrm{DIP}$ e aplicou-se 0,15 mg de Prostaglandina i.m.; em D9, aplicou-se $1 \mathrm{mg}$ de BE i.m. e em D10, aplicou-se $10 \mathrm{mg}$ i.m. de hormônio liberador de gonadotrofinas $\left(\mathrm{GnRH}^{3} ; 6\right.$ horas antes da IA) e procedeu-se a IA dos animais às 52 horas após a retirada do DIP.

- Protocolo eCG ( $n=32)$ : no D0 as fêmeas receberam DIP novo e aplicação i.m. de $2 \mathrm{mg} \mathrm{BE;} \mathrm{em} \mathrm{D8,} \mathrm{foi} \mathrm{feita} \mathrm{a} \mathrm{retirada} \mathrm{do} \mathrm{DIP,}$ aplicou-se 0,15 mg de Prostaglandina i.m. e 300 Unidades Internacionais $(\mathrm{UI})$ de gonadotrofina coriônica equina $\left(\mathrm{eCG}^{4}\right)$ i.m.; em D9, aplicou-se $1 \mathrm{mg}$ de BE i.m. e em; D10, aplicou-se i.m. $10 \mathrm{mg}$ de $\mathrm{GnRH}$ ( 6 horas antes da IA), e procedeu-se a IA dos animais às $52 \mathrm{~h}$ após a retirada do DIP.

O sêmen utilizado foi proveniente de central de inseminação artificial idônea e filiada à Associação Brasileira de Inseminação Artificial (ASBIA). Todas as inseminações foram realizadas pelo mesmo inseminador.

A taxa de gestação foi realizada aos 45 dias após a IATF. Os dados foram analisados através do teste de qui-quadrado.

Para a análise de custo de cada protocolo, foi utilizada metodologia proposta por Oliveira (2003), segundo modelo matemático abaixo:

Onde:

CIATF = custo da inseminação artificial em tempo fixo;

Ch $=$ custo dos hormônios;

Cds = custo da dose de sêmen;

$\boldsymbol{N}$ = número de fêmeas tratadas em cada protocolo;

Nia = número de inseminações realizadas;

$\mathbf{N p}$ = número de fêmeas prenhes ao diagnóstico de gestação.

\section{Resultados e discussão}

Como pode ser observado na Tabela 1, as taxas de prenhez encontradas foram de $42,6 \%, 45,5 \%$ e $68,8 \%$ para as vacas que receberam $\mathrm{BE}, \mathrm{GnRH}$ e eCG respectivamente. Os protocolos BE e eCG obtiveram diferença significativa $(p<0,05)$ nas taxas de prenhez, enquanto o GnRH não apresentou diferença significativa quando comparado aos demais.

Tabela 1: Variação na taxa de prenhez dos diferentes protocolos utilizados

\begin{tabular}{cccc}
\hline Protocolo & Total $(\mathbf{n})$ & Prenhas $(\mathbf{n})$ & \% de prenhez \\
\hline BE & 108 & 46 & $42,6^{*}$ \\
GnRH & 22 & 10 & $45,5^{n s}$ \\
eCG & 32 & 22 & $68,8^{*}$ \\
\hline
\end{tabular}

${ }^{*}$ Qui-quadrado significativo $(p<0,05 ; G l=2 ; n=162) e^{\text {ns }}$ - não significativo.

A ação dos estrógenos é independente do estágio do ciclo estral ou da onda de desenvolvimento folicular. A aplicação de estrógeno causa inicialmente uma supressão na secreção de hormônio folículo estimulante (FSH) e hormônio luteinizante

${ }^{3}$ Sincroforte ${ }^{\circledR}$, Ouro Fino Saúde Animal, Cravinhos, São Paulo, Brasil

${ }^{4}$ Sincroecg ${ }^{\circledR}$, Ouro Fino Saúde Animal, Cravinhos, São Paulo, Brasil 
(LH), levando à atresia dos folículos. Em consequência disso, segue um pico de $\mathrm{FSH}$ e recrutamento de uma nova onda de crescimento folicular (Binelli, 2006).

Segundo BURKE et al. (2003), foi observado que a emergência do folículo dominante pode ser atrasada devido ao uso de dosagens elevadas de BE no dia da inserção do implante intravaginal de progesterona. Resultados semelhantes foram encontrados por Moreira et al. (2007) quando compararam o uso do protocolo com BE e GnRH, apresentando $32 \%$ e $43 \%$ respectivamente. Já os resultados obtidos por Teixeira (2007) demonstraram que $48,15 \%$ dos animais tratados com $B E$ emprenharam contra $55,18 \%$ dos tratados com $\mathrm{GnRH}$.

A administração de análogos sintéticos ao $\mathrm{GnRH}$ exerce uma função fisiológica nas fêmeas, induzindo o pico pré-ovulatório de LH e consequentemente a ovulação e/ou luteinização do folículo, induzindo uma nova onda de crescimento folicular (Gottschall et al., 2008). Há estudos conflitantes sobre o uso do $\mathrm{GnRH}$ em vacas. Kaim et al. (2003) encontraram resultados variados sobre as taxas de prenhez em vacas submetidas a IATF após a aplicação de $\mathrm{GnRH}$. Os autores identificaram efeitos positivos do $\mathrm{GnRH}$ em primíparas, mas não em multíparas. Nesse estudo, animais com condição corporal inferior também foram beneficiadas pela aplicação do $\mathrm{GnRH}$, enquanto animais com condição corporal superior não foram favorecidos. Já Perry e Perry (2009) não encontraram efeitos favoráveis da aplicação do $\mathrm{GnRH}$ sobre as taxas de prenhez por ocasião da IATF em vacas e novilhas.

Taxas de prenhez superiores, com o uso do protocolo $\mathrm{GnRH}$, foram encontradas por Silva et al. (2004) com 48\%, Baruselli et al. (2002) obtiveram $59 \%$ e Vaca et al. encontraram $62 \%$. Em contrapartida, resultados inferiores foram encontrados por Gottschalll et al. (2010) com 40,6\%, Sá Filho et al. (2010) obtiveram 40,1\% e Souza et al. (2009) observaram apenas $28,9 \%$. Estes dados demonstram que os resultados encontrados com o uso de GnRH em protocolos de IATF ainda são contraditórios.

Segundo Baruselli et al. (2002) os baixos resultados de concepção provenientes da IATF estão relacionados com a incapacidade do folículo dominante culminar com o processo ovulatório. $\mathrm{O}$ pico pré-ovulatório de LH desencadeia a ovulação, que em vacas europeias ocorre quando o folículo adquire um diâmetro médio de $12 \mathrm{~mm}$. No momento do pico de $\mathrm{LH}$, a sincronia do crescimento folicular e o tamanho reduzido do folículo dominante são desafios a serem vencidos para possibilitar aumento das taxas de ovulação e prenhez na IATF (Sartori et al., 2001).

As vacas submetidas ao protocolo eCG obtiveram melhores resultados de prenhez que os demais. Resultados semelhantes foram encontrados por Sá Filho (2010), onde foi demonstrado que a eCG aumentou o diâmetro do folículo dominante, a taxa de ovulação, o diâmetro do corpo lúteo e consequentemente a taxa de prenhez. O tratamento com eCG no momento da retirada do implante de progesterona pode de gestação. ser uma ferramenta importante, uma vez que a eCG possui a singularidade de possuir dupla ação (FSH e LH) na mesma molécula.

Estudos realizados por outros autores também encontraram melhores taxas de prenhez com o uso da eCG. Os resultados do presente trabalho, corroboram aos de Baruselli et al. (2008), Souza (2008), e Penteado et al. (2004), onde obtiveram prenhez de $63,2 \% ; 38,25 \%$ e $55,6 \%$ com uso da eCG e $41,7 \% ; 19,8 \%$ e $42,2 \%$ sem eCG respectivamente.

Desta forma, o tratamento com eCG é recomendado quando uma grande proporção dos animais se encontra em anestro, ou com baixo escore de condição corporal. Existem muitos relatos de aumento na fertilidade após a incorporação da eCG em protocolos de IATF. A melhora na taxa de concepção em animais que receberam eCG na retirada do dispositivo intravaginal de progesterona, pode estar relacionada com o aumento da taxa de ovulação de animais em anestro e aos maiores níveis de progesterona circulante no diestro subsequente, minimizando falhas no reconhecimento embrionário da gestação (Baruselli et al., 2004). Portanto, a adição de eCG no momento da remoção do implante de progesterona, é uma alternativa para aumentar as taxas de prenhez em programas de IATF, sobretudo em vacas em anestro ou com baixas condições corporais (Bó et al., 2004).

A análise econômica foi realizada a partir da média dos preços praticados na região dos hormônios utilizados e considerando também o valor médio das doses de sêmen (Tabela 2). Uma vez que a margem de lucro estimada no valor do litro de leite é aproximadamente $10 \%$, e que a produção média das vacas utilizadas no experimento foi de 10L/vaca/dia, tem-se lucro de $\mathrm{R} \$ 0,85$ por dia de lactação. Portanto, dividindo-se o custo total do protocolo utilizado pelo lucro estimado, tem-se o resultado em dias de lactação que cobrem os custos empregados na utilização do protocolo de IATF.

Portanto, como pode ser observado na Tabela 2, foram necessários aproximadamente 39,53 e 50 dias de lactação para cobrir os custos dos protocolos BE, GnRH e eCG respectivamente, considerando-se uma produtividade média de 10L/vaca/dia.

Tabela 2: Análise econômica dos protocolos utilizados

\begin{tabular}{cccc}
\hline Protocolo & $\begin{array}{c}\text { Custo da inseminação artificial em } \\
\text { tempo fixo (CIATF) } \\
\{(\text { Ch } \times N)+(C d s \times N i a)\}\end{array}$ & $\begin{array}{c}\text { Valor final do } \\
\text { protocolo }(\mathrm{R} \$)\end{array}$ & $\begin{array}{c}\text { Dias de lactação para } \\
\text { cobrir os custos do } \\
\text { protocolo utilizado }\end{array}$ \\
\hline BE & $\frac{\{(13,71 \times 108)+(25 \times 1)\}}{46}$ & 32,73 & 38,50 \\
\hline GnRH & $\frac{\{(19,32 \times 22)+(25 \times 1)\}}{10}$ & 45,00 & 52,94 \\
\hline eCG & $\frac{\{(28,50 \times 32)+(25 \times 1)\}}{22}$ & 42,59 & 50,10 \\
\hline
\end{tabular}

$\boldsymbol{C h}=$ custo dos hormônios; $\boldsymbol{C} \boldsymbol{d} \boldsymbol{s}=$ custo da dose de sêmen; $\boldsymbol{N}=$ número de fêmeas tratadas em cada protocolo; $\boldsymbol{N i a}=$ número de inseminações realizadas; $\boldsymbol{N p}=$ número de fêmeas prenhes ao diagnóstico

O protocolo com retorno financeiro mais eficiente é o $B E$, seguido do eCG e por fim o $\mathrm{GnRH}$. Neste contexto, vale ressaltar que o protocolo mais econômico financeiramente foi o $\mathrm{BE}$, em 
contrapartida foi o que apresentou menores índices de prenhez. O protocolo que se apresentou mais oneroso foi o $\mathrm{GnRH}$, pois este é relativamente caro quando comparado ao $\mathrm{BE}$, e as taxas de prenhez apresentadas pelo mesmo não foram suficientes para reduzir seu preço final. Já o protocolo eCG, apresentou maior despesa em relação aos custos hormonais. No entanto, levando-se em consideração a sua taxa de prenhez, o número de animais utilizados e o número de vacas prenhas, pôde-se minimizar as despesas com os hormônios, quando comparado ao protocolo $\mathrm{GnRH}$.

Uma vez que as principais limitações da bovinocultura seja devido ao prolongado anestro pós-parto e falhas na detecção do estro (Yavas \& Walton, 2000), o emprego da IATF pode contornar estes problemas, pois os protocolos hormonais podem ser utilizados em vacas em anestro e assim, promover a regulação da cascata hormonal e a sincronização da ovulação e, deste modo, restabelecer a liberação dos hormônios responsáveis para o retorno da ciclicidade e de suas funções reprodutivas.

\section{Conclusões}

O protocolo eCG apresentou maior custo mas, em contrapartida, foi o que apresentou melhores resultados de prenhez. O protocolo BE é o de menor custo econômico, no entanto, é o que apresenta menores taxas de prenhez.

\section{Agradecimentos}

Ao Programa RIO GENÉTICA por permitir a reprodução de parte parcial dos resultados apresentados no presente estudo.

\section{Referências}

BARUSELLI, P.S.; MADUREIRA, E.H.; SILVA, R. Efeito de diferentes protocolos de IATF na eficiência reprodutiva de vacas de corte lactantes. Revista Brasileira de Reprodução Animal, v. 26, n. 3, p. 218-221, 2002.

BARUSELLI, P.S.; JACOMINI, J.O.; SALES, J.N.S. Importância do emprego da eCG em protocolos de sincronização para IA, TE e SOV em tempo fixo. In: III SIMPÓSIO INTERNACIONAL DE REPRODUÇÃO ANIMAL APLICADA, v. 1, p. 146-167, 2008.

BARUSELLI, P. S.; MADUREIRA, E. H.; SÁ FILHO, M.F. Efeito do tratamento com eCG conforme o escore de condição corporal na taxa de concepção de vacas Nelore inseminadas em tempo fixo. Acta Scientiae Veterinarie, v. 32, p. 228, 2004.

BINELLI, M.; IBIAPINA, B.T.; BISINOTTO, R.S. Bases fisiológicas, farmacológicas e endócrinas de sincronização de crescimento folicular e da ovulação. Acta Scientiae Veterinariae, v. 34, p. 1-7, 2006.

BÓ, G. A.; BROGLIATTI, G. M.; PIERSON, R. A. Local versus systemic effect of exogenous estradiol-17 $\beta$ on ovarian follicular dynamics in heifers with progestogen implants. Animal Reproduction Science, v. 59, p. 141-157, 2004.

BURKE, C. R.; MUSSARD, M. L.; GRUM, D. E. Effects of maturity of the potencial ovulatory follicle on induction of oestrus and ovulation in cattle with oestradiol benzoate. Animal Reproduction Science, v. 66, p.161-174, 2003.

COLBERT, M. Indústria Leiteira Fluminense. <http://www.rj.gov.br/ web/imprensa/exibeconteudo?article-id=791379>, acessado em 6 de junho de 2012.

EDMONSON, A.J.; LEAN, I.J.; WEAVER, L.D.; FARVER, L. D.; WEBSTER, G. A body condition scoring chart for Holstein dairy cows. Journal Dairy Science, v.72, n.1, p. 68-78, 1989.

FAERJ/SEBRAE-RJ. Diagnóstico da cadeia produtiva do Leite do Estado do Rio de Janeiro - Federação de Agricultura, Pecuária e Pesca do Estado do Rio de Janeiro - FAERJ e Serviço de Apoio às Micro e Pequenas Empresas do Estado do Rio de Janeiro SEBRAE/RJ, 264 p., Rio de Janeiro, 2003.

FERREIRA, A. M., Interação Nutrição e Reprodução: Manejo Reprodutivo de Fêmeas nos Trópicos. In: ANAIS II SIMPÓSIO DE PRODUÇÃO DE GADO DE CORTE, p. 137-146, 2000.
GOTTSCHALL, C.S.; SCHULER, M.V.; MARTINS, C.T.D.C.; ALMEIDA, M.R.; MAGERO, J.; SOARES, J.C.R. Efeitos do uso do $\mathrm{GnRH}$ no momento da IATF e dias pós-parto sobre a taxa de prenhez em vacas de corte com cria ao pé. Veterinária em Foco, v. 7, p. 124-134, 2010.

INSTITUTO BRASILEIRO DE GEOGRAFIA E ESTATÍSTICA. Banco de dados agregados. Disponível em: <http://www.ibge. gov.br/home/presidencia/noticias/noticia_visualiza.php?id_ noticia=1464\&id_pagina=1>. Acesso em: 10 junho 2012.

KAIM, M.; BLOCH, A.; WOLFENSON, D. Effects of GnRH at the onset of estrus on timing of ovulation, endocrine responses, and conception. Journal of Dairy Science, v. 86, p. 2012-2021, 2003.

MONTEZUMA JR, P. A. Desempenho reprodutivo pós-parto de vacas leiteiras mestiças (3/8 Holandês X 5/8 Gir) submetidas a um tratamento hormonal à base de $\mathrm{GnRH}$ e prostaglandina $\mathrm{F} 2 \alpha$. 2001. 70F. Dissertação (Mestrado) - Universidade Federal do Ceará, Fortaleza, 2001.

MOREIRA, R.F.C.; MADUREIRA, E.H.; BINELLI, M.; GONÇALVES, J.R.; LIMA, L.G. Uso do protocolo Crestar® em tratamentos utilizando benzoato de estradiol, PGF2 $\alpha$, PMSG e GnRH para controle do ciclo estral e ovulação em vacas de corte. Brazilian Journal Research Animal Science, v. 44, p. 56-62, 2007.

OLIVEIRA, K.P.L. Comparação de dois métodos de indução e sincronização do estro e da ovulação em vacas leiteiras mestiças no anestro pós parto. Dissertação (Mestrado), 66 f., 2003.

PENTEADO, L.; AYRES, H.; REIS, E.L.; MADUREIRA, E.H.; BARUSELLI, P.S. Efeito do eCG e do desmame temporário na taxa de prenhez de vacas Nelore lactantes inseminadas em tempo fixo. In: XVIII REUNIÃO ANUAL DA SOCIEDADE BRASILEIRA DE TRANSFERÊNCIA DE EMBRIÕES. Acta Scientiae Veterinariae, v. 32, p. 223, 2004.

PERRY, G.A.; PERRY, B.L. GnRH treatment at artificial insemination in beef cattle fails to increase plasma progesterone concentratios or pregnancy rates. Theriogenology, v. 71, p. 775779, 2009.

SÁ FILHO, M.F.; TORRES-JUNIOR, J.R.; PENTEADO, L.; GIMENES, L.U.; FERREIRA, R.M.; AYRES, H.; SALES, J.N. Equine chorionic gonadotropin improves the efficiency of a progestagen-based timed-artificial insemination protocol in nelore (Bos indicus) heifers. Animal Reproduction Science, v. 118, p. 182-187, 2010. 
SÁ FILHO, O. G.; DIAS, C. C.; VASCONCELOS, J. L. M. Effect of progesterone or $17 \beta$ - estradiol on luteal lifespan in anoestrous Nelore cows. Journal Animal Science, v. 84, suppl. 1, p. 207 (Abstract), 2006.

SARTORI, R., FRICKE, P. M., FERREIRA, J. C. Follicular deviation and acquisition of ovulatory capacity in bovine follicles. Biology Reproduction, v.65, p.1403-1409, 2001.

SILVA, R.C.P.; RODRIGUES, C.A.; MARQUES, M.O.; AYRES, H.; REIS, E.L.; MADURE, E.H.; BARUSELLI, P.S. Efeito do eCG e $\mathrm{GnRH}$ na taxa de prenhez de vacas nelore lactantes inseminadas em tempo fixo. Acta Scientiae Veterinariae, v.32, p. 221, 2004.

SOUZA, A. H.; VIECHNIESKI, S.; LIMA, F.A.; SILVA, F.F.; ARAUJO,R.; BÓ, G.A.; WILTBANK, M.C.; BARUSELLI, P.S. Effects of equine chorionic gonadotropin and type of ovulatory stimulus in timed-IA protocol on reproductive responses in dairy cows. Theriogenology, v. 72, p. 10-21, 2009.
SOUZA, A. H. Inseminação Artificial em Tempo Fixo em Vacas Holandesas de Alta Produção. 152 f., Tese (Doutorado), 2008 Faculdade de Medicina Veterinária e Zootecnia, Universidade de São Paulo, 2008.

TEIXEIRA, L. Utilização de GnRH como efeito somatório na indução de ovulação de bovinos, 22f. Tese (Pós-graduação). 2007.

VACA, R.J.A.; PRANDO, A.; BALDO, A.; LA SOTA, R.L. Tasa de preñez luego de la utilización de benzoato de estradiol 24 horas antes o GnRH en el momento de la IATF en vacas multíparas para carne. In: VII SIMPOSIO INTERNACIONAL DE REPRODUCCIÓN ANIMAL. Córdoba. p.268. 2007.

YAVAS, Y.; WALTON, J.S. Postpartum acyclicity in suckled beef cows: a review. Theriogenology, v. 54, p. 25-55, 2000. 